\title{
An Approach for Automatic Voice Signal Detection (AVSD) Using Matlab
}

\author{
Shiv Kumar, Member, IACSIT, IAENG Aditya Shastri and R.K. Singh
}

\begin{abstract}
In this paper titled: "Automatic Voice Signal Detection (AVSD)", is a technique which identify constitutional and unconstitutional voice signal automatically. AVSD is performed on the basis of frequency content of voice signal for several samples of the same sound. An underline purpose of this paper is to investigate automatically fake voice signal in the security system. Frequency content is mapped to the frequency domain by computing its DFT using the FFT algorithm. The voice signal detection algorithm is formulated by continually computing the difference of the absolute average of two adjacent voice windows, and comparing it to a predefined threshold.
\end{abstract}

Index Terms-Algorithm, Frequency Content, Threshold, FFT, Window

\section{INTRODUCTION}

Human beings can here the frequency range approximately from $20 \mathrm{~Hz}$ to $20 \mathrm{KHz}$. If a frequency that is very close to $20 \mathrm{~Hz}$ to $20 \mathrm{KHz}$, could assume that for hearing this closed signal becomes more difficult. Human auditory systems have a perceptual property that may be auditory threshold of hearing (ATH) or auditory masking [5-9]. When a strong signal makes a temporal or spectral neighborhood of weaker signal minute, this means that masking effect can be observed in the time and frequency domain. If two signals occur at the same time and one signal is masked by another signal than this is called simultaneous masking or frequency masking. A weak signal emitted after the end of louder signal and masked by louder signal.

By different news channel and different Media we generally come to know that a person is denying from the statement which may or may not be given by him, by saying that this is not his voice. We thought for above problem and finally decided to develop an approach so that we will be able to find whether the person is innocent or not. AVSD is an approach to find-out whether a person is denying from his/her statement or not mean distinguish between the constitutional and unconstitutional voice signal.

Voice signal detection is becoming increasingly popular and can be found in luxury cars, mobile phones, dictation office software, toys, and appliances. It is defined as the

Manuscript received October 9, 2010.

Shiv Kumar is an Asst. Professor, Department of Information Technology at Technocrat Institute of Technology-Bhopal (M.P.)-462021, India (Mobile No.: +91-9669981618, E-mail: shivksahu@rediffmail.com, website: www.shivksahu.webs.com).

Prof. Aditya Shastri is Vice Chancellor of Banasthali University, Tonk (Rajasthan)-304022, India (Phone: +91-1438-228373, 228787, E-mail: adityashastri@yahoo.com).

Prof. R.K. Singh is Vice Chancellor of ITM University, Gurgaon (Haryana), India (Phone: +91-124-2365811, 2365812, E-mail: vcrks@rediffmail.com). ability to identify a spoken word or a sequence of words.

\section{A. Wavelet Theory}

The fundamental idea behind wavelets is to analyze according to the scale. Indeed, some researchers in the wavelet field feel that, by using wavelets, one is adopting a whole new mindset or perspective in processing data. Wavelets are functions which satisfy certain mathematical requirements and are used in representing data or other functions. Approximation using superposition of functions has existed since the arly 1800's, when Joseph Fourier discovered that he could superpose sines and cosines to represent other functions. However, in wavelet analysis, the scale that one uses for looking at data plays a vitae role. Wavelet algorithms process data at different scales or resolutions. If we look at a signal with a large "window," we would notice gross features. Similarly, if we look at a signal with a small "window," we would notice small discontinuities [10-11].

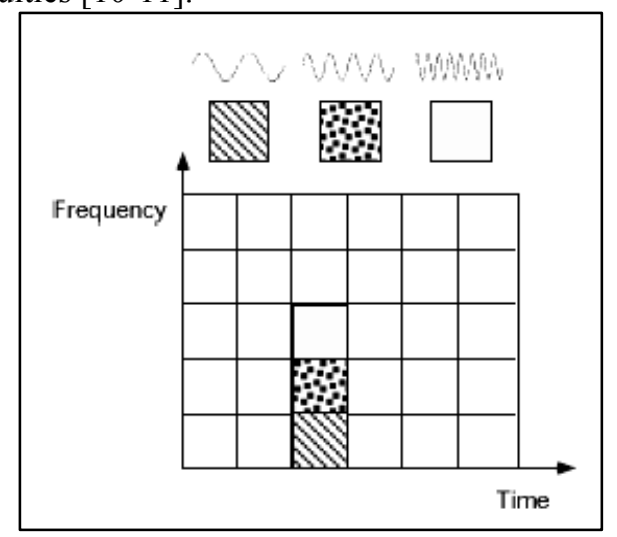

Fig. 1: Fourier basis functions, time-frequency tiles and coverage of the Time-frequency plane

Fig. 1 shows a windowed Fourier transform, where the window is simply a square wave [10-11]. The square wave window truncates the sine or cosine function to fit a window of a particular width. Because a single window is used for all frequencies in the WFT, the resolution of the analysis is same at all locations in the time-frequency plane.

Now, the question is why these features make wavelets interesting and useful? For many decades, scientists have wanted more appropriate functions than the sines and cosines which comprise the bases of Fourier analysis, to approximate choppy signals. According to their definition, these functions are non-local (and stretch out to infinity), and therefore do a very poor job in approximating sharp spikes. But with wavelet analysis, we can use approximating functions that are contained neatly in finite domains. Wavelets are well-suited for approximating data with sharp discontinuities.

The wavelet analysis procedure is to adopt a wavelet 
prototype function, called an "analyzing wavelet" or "mother wavelet." Temporal analysis is performed with a contracted, high-frequency version of the prototype wavelet, while frequency analysis is performed with a dilated, low-frequency version of the prototype wavelet. Because the original signal or function can be represented in terms of a wavelet expansion (using coefficients in a linear combination of the wavelet functions), data operations can be performed using just the corresponding wavelet coefficients. And if we further choose the best wavelets adapted to our data, or truncate the coefficients below a threshold, our data is sparsely represented. This "sparse coding" makes wavelets an excellent tool in the field of data compression.

Wavelet can also apply in other fields like [10-11] Astronomy, Acoustics, Nuclear Engineering, Sub-band Coding, Signal and Image Processing, Neurophysiology, Music, Magnetic, Resonance Imaging, Speech Discrimination, Optics, Fractals, Turbulence, EarthquakePrediction, Radar, Human Vision, Pure Mathematics Applications.

\section{B. Historical Perspective}

In the 1930s, several groups working independently did research in the representation of functions by using scale-varying as the basic functions. Understanding the concepts of basis functions and scale-varying basis functions is keys to understanding wavelets.

By using a scale-varying basis function called the Haar basis function Paul Levy, a 1930s physicist, investigated Brownian motion, a type of random signal. He found the Haar basis function superior to the Fourier basis functions for studying small complicated details in the Brownian motion. Another 1930s research effort by Little-wood, Paley, and Stein involved computing the energy of a function $\mathrm{f}(\mathrm{x})$ [10-11] which is given in equation-1.

$$
\text { Energy }=\frac{1}{2}\left[\int|f(x)|^{2}\right] \mathrm{d} x \ldots \ldots \ldots . .(1)
$$

The computation produced different results if the energy was concentrated around few points or distributed over a larger interval. This result disturbed the scientists because it indicated that energy might not be conserved. The researchers discovered a function that can vary in scale and can conserve energy when computing the functional energy.

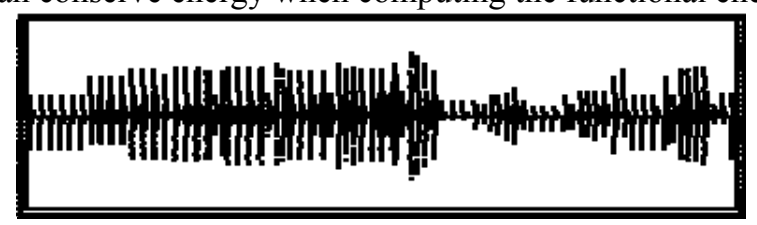

Fig. 2: Wavelets for musical tone

It is simpler to explain a basis function if we move out of the realm of analog (functions) and into the realm of digital (vectors). Every two-dimensional vector $(\mathrm{x} ; \mathrm{y})$ is a combination of the vector $(1 ; 0)$ and $(0 ; 1)$ : These two vectors are the basis vectors for $(\mathrm{x} ; \mathrm{y})$ : Why? Notice that $\mathrm{x}$ multiplied by $(1 ; 0)$ is the vector $(x ; 0)$; and y multiplied by $(0 ; 1)$ is the vector $(0 ; y)$ : The sum is $(x ; y)$. The best basis vectors have the valuable extra property that the vectors are perpendicular, or orthogonal to each other. For the basis $(1 ; 0)$ and $(0 ; 1)$; this criteria is satisfied [11].
Now let's go back to the analog world, and see how to relate these concepts to basis functions. Instead of the vector (x; $y)$; we have a function $f(x)$ : Imagine that $f(x)$ is a musical tone, say the note A in a particular octave. We can construct A by adding sines and cosines using combinations of amplitudes and frequencies. The sines and cosines are the basis functions in this example, and the elements of Fourier synthesis. For the sines and cosines chosen, we can set the additional requirement that they be orthogonal. How? By choosing the appropriate combination of sine and cosine function terms whose inner product add up to zero. The particular set of functions that are orthogonal and that construct $\mathrm{f}(\mathrm{x})$ are our orthogonal basis functions for this problem.

Now we will look at Scale-Varying a basic function that varies in scale by chopping up the same function or data space using different scale sizes. For example, imagine we have a signal over the domain from 0 to 1 . We can divide the signal with two step functions that range from 0 to $1 / 2$ and $1 / 2$ to 1 . Then we can divide the original signal again using four step functions from 0 to $1 / 4,1 / 4$ to $1 / 2,1 / 2$ to $3 / 4$, and $3 / 4$ to 1 . And so on. Each set of representations code the original signal with a particular resolution or scale.

Victor Wickerhauser has suggested that wavelet packets could be useful in sound synthesis. His idea is that a single wavelet packet generator could replace a large number of oscillators. Through experimentation, a musician could determine combinations of wave packets that produce especially interesting sounds. Wickerhauser feels that sound synthesis is a natural use of wavelets. Say one wishes to approximate the sound of a musical instrument. A sample of the notes produced by the instrument could be decomposed into its wavelet packet coefficients. Reproducing the note would then require reloading those coefficients into a wavelet packet generator and playing back the result. Transient characteristics such as attack and decay roughly, the intensity variations of how the sound starts and ends- could be controlled separately (for example, with envelope generators), or by using longer wave packets and encoding those properties as well into each note. Any of these processes could be controlled in real time, for example, by a keyboard [11].

Notice that the musical instrument could just as well be a human voice, and the notes words or phonemes. A wavelet-packet-based music synthesizer could store many complex sounds efficiently because:

- Wavelet packet coefficients, like wavelet coefficients, are mostly very small for digital samples of smooth signals.

- And discarding coefficients below a predetermined cutoff introduces only small errors when we are compressing the data for smooth signals.

Similarly, a wave packet-based speech synthesizer could be used to reconstruct highly compressed speech signals. Fig.-2 illustrates a wavelet musical tone or tone-burst [11-12].

\section{Wavelet Application}

Wavelets have scale aspects and time aspects; consequently every application has scale and time aspects. To 
clarify them we try to untangle the aspects somewhat arbitrarily.

For scale aspects, there is one idea around the notion of local regularity. For time aspects, there is a list of domains. When the decomposition is taken as a whole, the de-noising and compression processes are the center points [3, 13-19].

As a complement to the spectral signal analysis, new signal forms appear. They are less regular signals than the usual ones. The cusp signal presents a very quick local variation. Its equation is $\mathrm{t}^{\mathrm{r}}$ with $\mathrm{t}$ close to 0 and $\mathrm{o}<\mathrm{r}<1$. The lower $\mathrm{r}$ is sharper than the signal. To illustrate this notion physically, imagine we take a piece of aluminum foil. The surface is very smooth, very regular. We first crush it into a ball, and then spread it out so that it looks like a surface. The asperities are clearly visible. Each one represents a two-dimension cusp and analog of the one dimensional cusp. If we crush again the foil more tightly in a more compact ball when we spread it out, the roughness increases and the regularity decrease.

Several domains use the wavelet techniques of regular study:

- Biology for cell membrane recognition, to distinguish the normal from the Pathological membranes.

- Metallurgy for the characterization of rough surfaces.

- Finance (which is more surprising), for detecting the properties of quick variation of values.

- In Internet traffic description, for designing the services size.

Let's switch to time aspects. The main goals are:

- Rupture and edges detection.

- Study of short-time phenomena as transient processes.

As domain applications, we get:

- Industrial supervision of gear-wheel.

- Checking undue noises in craned or dented wheels, and more generally in nondestructive control quality processes.

- Detection of short pathological events as epileptic crises or normal ones as evoked potentials in EEG (medicine).

- SAR imagery.

- Automatic target recognition.

- Intermittence in physics.

\section{Wavelet Vs. Fourier Analysis}

In the well-known Fourier analysis, signal is broken down into constituent sinusoids of different frequencies. Another way to think of Fourier analysis is as a mathematical technique for transforming our view of the signal from
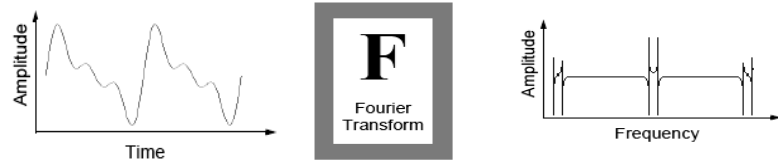

Fig. 3: Fourier Transform from time domain to frequency

time-based to frequency-based. A simple Fourier transform [10-11] is illustrated in Fig.-3.Taking the Fourier transform of a signal can be viewed as a rotation in the function space of the signal from the time domain to the frequency domain.
Similarly, the wavelet transforms can be viewed as transforming the signal from the time domain to wavelet domain. This new domain contains more complicated basis functions called wavelets, mother wavelets or analyzing wavelets. A simple Fourier transform [10] is illustrated in Fig. 4.
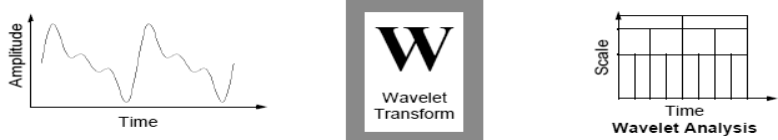

Fig. 4: Wavelet Transform from time domain to wavelet domain]

Mathematically, the process of Fourier analysis is represented by the Fourier transform which is given in equation-2.

$$
F(\omega)=\int_{-\omega}^{\omega} f(t) e^{-j \omega t} d t
$$

Which is the sum over all time of the signal $\mathrm{f}(\mathrm{t})$ multiplied by a complex exponential. The results of the transform are the Fourier coefficients $F(\omega)$, which when multiplied by a sinusoid of frequency $\omega$ yield the constituent sinusoidal components of the original signal.

Similarly, the continuous wavelet transform (CWT) [10, 22 ] is defined as the sum over all time of the signal multiplied by scaled, shifted versions of the wavelet function $(\psi)$ which is given in equation-3.

$$
C(\text { Scale }, \text { Position })=\int_{-\infty}^{\infty} f(t) \Psi(\text { Scale }, \text { Position, } t) d t \text {........(3) }
$$

The results of the CWT are many wavelet coefficients C, which is a function of scale and position. Multiplying each coefficient by the appropriately scaled and shifted wavelet yields the constituent wavelets of the original signal.

The basis function in both Fourier and wavelet analysis are localized in frequency making mathematical tools such as power spectra (power in a frequency interval) useful at picking out frequencies and calculating power distributions. The most important difference between these two kinds of transforms is that individual wavelet functions are localized in space. In contrast Fourier sine and cosine functions are non-local and are active for all time $t$.

This localization feature, along with wavelets localization of frequency, makes many functions and operators using wavelets "sparse". When transformed into the wavelet domain. This sparseness, in turn results in a number of useful applications such as data compression, detecting features in images and de-noising signals.

A major draw back of Fourier analysis is that in transforming to the frequency domain, the time domain information is lost. When looking at the Fourier transform of a signal, it is impossible to tell when a particular event took place. In an effort to correct this deficiency, Dennis Gabor (1946) adapted the Fourier transform to analyze only a small section of the signal at a time. This technique is called windowing the signal. Gabor's adaptation, called the Short-Time Fourier Transform (STFT) or Windowed Fourier Transform (WFT) that maps a signal into a two-dimensional function of time and frequency i.e. WFT gives information about signals simultaneously in the time domain and in the frequency domain. 

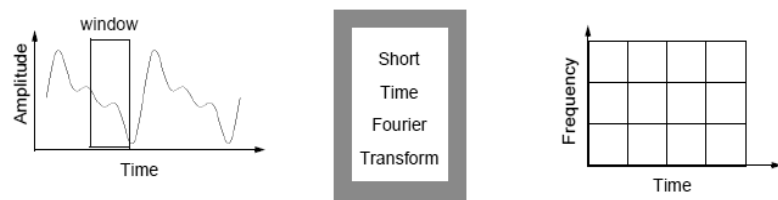

Fig. 5: Windowing the signal into two dimensional function of time and frequency

Fig.-5 represents a sort of compromise between the time and frequency based views of a signal [10]. It provides some information about both when and at what frequencies a signal event occurs. However, we can only obtain this information with limited precision, and that precision is determined by the size of the window. While the STFT compromise between time and frequency information can be useful, the drawback is that if once choose a particular size for the time window, that window is same for all frequencies. Many signals require a more flexible approach in which we can vary the window size to determine more accurately either time or frequency. To illustrate the time-frequency resolution differences between the Fourier transform and the wavelet transform consider the Fig. 6 that show a Windowed Fourier Transform, where window is simply a square wave. The square wave window truncates the sine or cosine function to fit a window of a particular width. Because a single window is used for all frequencies in the WFT, the resolution of the analysis is the same at all locations in the time frequency plane.

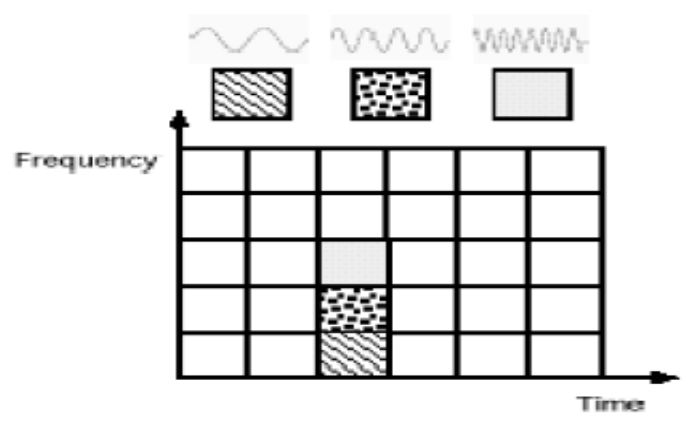

Fig. 6: WFT or STFT Resolution

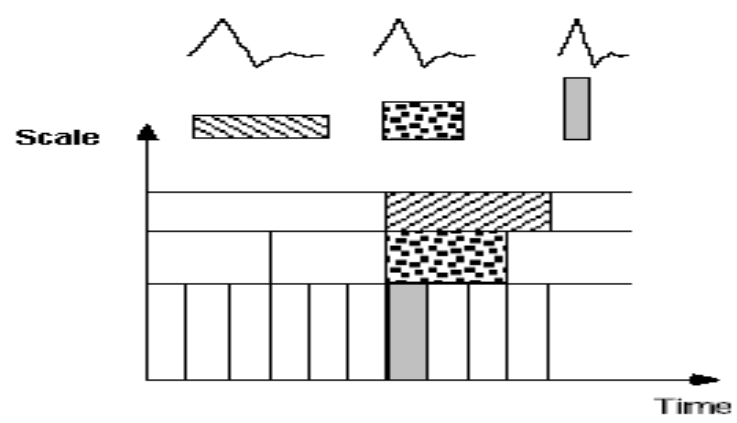

Fig. 7: Wavelet Resolution

An advantage of wavelet transforms is that the windows vary. Wavelet analysis allows the use of long time intervals where we want more precise low-frequency information, and shorter regions where we want high-frequency information. A way to achieve this is to have short high-frequency basis functions and long low-frequency ones.

Fig.-7 shows a time-scale view for wavelet analysis rather than a time frequency region [10]. Scale is inversely related to frequency. A low-scale compressed wavelet with rapidly changing details corresponds to a high frequency. A high-scale stretched wavelet which is slowly changing has a low frequency.

\section{E. Discrete Wavelet Transform}

The Discrete Wavelet Transform (DWT) [10, 20-22] involves choosing scales and positions based on powers of two so called dyadic scales and positions. The mother wavelet is rescaled or dilated by powers of two and translated by integers. DWT is defined by equation- 4 .

$$
W(j, k)=\sum_{j} \sum_{k} x(k) 2^{-\frac{j}{2}} \psi\left(2^{-j} n-k\right) \ldots \ldots
$$

Where $\psi(t)$ is a time function with finite energy and fast decay called the mother wavelet. The DWT analysis can be performed using a fast, pyramidal algorithm related to multi-rate filter-banks.

Specifically, a function $f(t) \epsilon L^{2}(R)$ (defines space of square integral functions) can be represented as per equation-5.

$$
f(t)=\sum_{j=1}^{L} \sum_{k=-\infty}^{\infty} d(j, k) \psi\left(2^{-j} t-k\right)+\sum_{k=-\infty}^{\infty} a(L, k) \phi\left(2^{-L} t-k\right) \ldots .(5)
$$

The function $\psi(t)$ is known as mother wavelet, while $\phi(t)$ is known as the scaling function. The set of following function:

$$
\left\{\sqrt{2^{-l}} \phi\left(2^{-L} t-k\right), \sqrt{2^{-j}} \psi\left(2^{-L} t-k\right) \mid j \leq L, j, k, L \in Z\right\}
$$

Where $\mathrm{Z}$ is the set of integers is an orthonormal basis for $\mathrm{L}^{2}(\mathrm{R})$. The numbers $a(L, k)$ are known as the approximation coefficients at scale $\mathrm{L}$, while $d(j, k)$ are known as the detail coefficients at scale $\mathrm{j}$.

The approximation and detail coefficients can be expressed as per equation- 6 and 7 .

$$
\begin{aligned}
& a(L, k)=\frac{1}{\sqrt{2^{L}}} \int_{-\infty}^{\infty} f(t) \phi\left(2^{-L} t-k\right) d t . \\
& a(j, k)=\frac{1}{\sqrt{2^{j}}} \int_{-\infty}^{\infty} f(t) \psi\left(2^{-j} t-k\right) d t .
\end{aligned}
$$

To provide some understanding of the above coefficients consider a projection $f_{l}(t)$ of the function $f(t)$ that provides the best approximation (in the sense of minimum error energy) to $f(t)$ at a scale $l$. This projection can be constructed from the coefficients $a(L, k)$ by using equation-8.

$$
f_{1}(t)=\sum_{k=-\infty}^{\infty} a(l, k) \phi\left(2^{-l} t-k\right) \ldots \ldots \ldots
$$

As the scale $l$ decreases, the approximation becomes finer, converging to $f(t)$ as $l \rightarrow 0$. The difference between the approximation at scale $l+1$ and that at $l, f_{l+1}(t)-f_{l}(t)$, is completely described by the coefficients $d(j, k)$ using the equation-9.

$$
f_{l+1}(t)-f_{1}(t)=\sum_{k=-\infty}^{\infty} d(l, k) \psi\left(2^{-l} t-k\right) \ldots
$$


Using these relations, given $a(L, k)$ and $\{d(j, k) \mid j \leq L\}$, it is clear that we can build the approximation at any scale. Hence, the wavelet transform breaks the signal up into a coarse approximation $f_{L}(t)$ (given $a(L, k)$ ) and a number of layers of detail $\left\{f_{j+l}(t)-f_{j}(t) \mid j<L\right\}$ (given by $\{d(j, k) \mid \mathrm{j} \leq \mathrm{L}\}$ ). As each layer of detail is added, the approximation at the next finer scale is achieved.

\section{Problem IdentificAtion AND Literature SurVey}

Mr. Shaleena Jeeawoody [1] found whether could develop a more efficient car security system using the technology voice recognition. The function of this voice recognition car security system is to unlock only when it recognizes a password spoken by the password holder. As per result shows that among the 15 words tested, no two voices overlapped. For a given word, the voice spectrum differs from one person to another. Work was good but what about if security is protected more than 15 words. To solve this type problem, AVSD is approached to protect the system for more than 15 words.

Mr. Khalid Saeed [2] explained voice recognition where it is not defined that which type file will recognize and how? In this research work AVSD is for practical approach where all the problems will resolve and .wav file is used for voice detection.

Maria Markaki [3] explained a novel feature set for the detection of singing voice in old and new musical recordings but what about if fake voice is recorded for voice detection. To solve this type problem, AVSD is approached.

Vijay K Chaudhari [4] developed Voice Spectrum Analysis for fake voice is done manually with the help of various DSP parameters where .wav is used which take more time for processing because all the work is related manually.
AVSD is an approach where fake voice detection will do automatically.

\section{METHODOLOGY}

\section{A. Block Diagram for AVSD}

The system must know when a voice signal is input. Thus, a detection algorithm has been devised. This is done by continually computing the difference of the absolute average of two adjacent sound windows (sets of consecutive sound data), and comparing it to a predefined threshold. The detector algorithm can be broken down as per following steps:

1) The absolute average $w_{1}$ of a sound window of length $W$ is computed from the sound samples $s_{1}$ starting at $s_{a}$ and ending at $\mathrm{s}_{\mathrm{b}}$ as shown in equation- 10 .

$$
w_{1}=\frac{1}{w} \sum_{i=a}^{b}\left|s_{i}\right|
$$

2) The average of the second window w2 is computed from the sound samples si starting at $\mathrm{sb}$ and ending at sc as shown in equation-11.

$$
w_{2}=\frac{1}{w} \sum_{i=b}^{c}\left|s_{i}\right|
$$

3) The difference between $\mathrm{w}_{2}$ and $\mathrm{w}_{1}$ is compared to the threshold value Th. If it is larger, the spoken word is considered to start at $s_{c}$ else, the algorithm goes on to step 4.

4) The average of the oldest window $\left(\mathrm{w}_{1}\right)$ is discarded, and replaced by $\mathrm{w}_{2}$. Then, the algorithm goes back to step 2 .

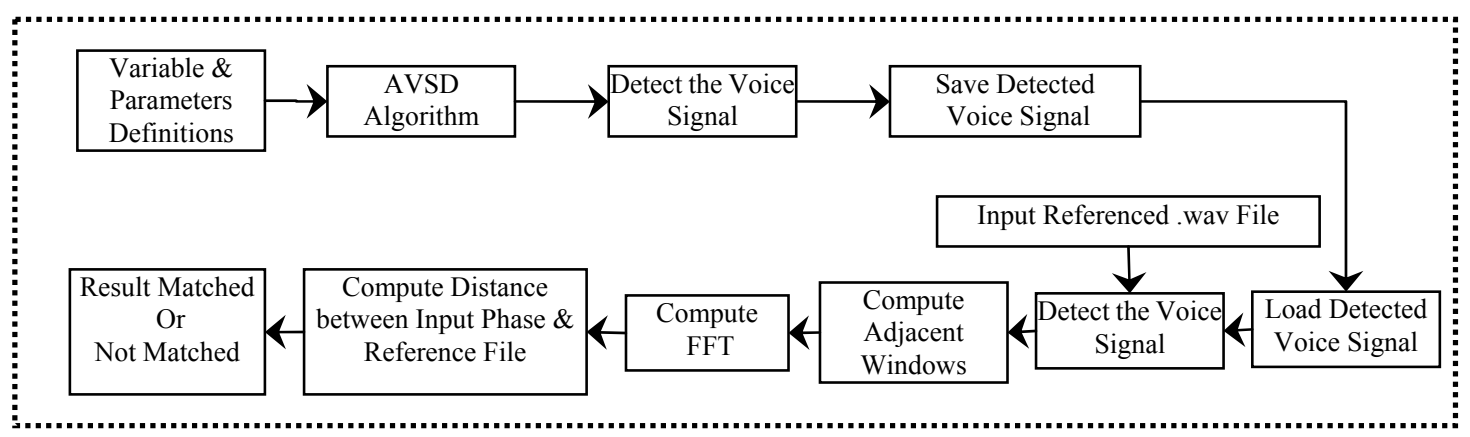

Fig. 8: Block Diagram for AVSD

Once the voice is detected, it is mapped to the frequency domain by computing its Discrete Fourier Transform (DFT) using the Fast Fourier Transform (FFT) algorithm. The sound is sampled at $5 \mathrm{kHz}$, five 1024-points FFTs are required to fully characterize a single word.

The comparison between input voice and the reference voice is done by taking the Euclidean distance between them. To do this, they are considered as five 1024-dimensional vectors (one for each matrix row), and the average of their respective Euclidean distance is computed. This is shown in equation-12, where $\mathrm{D}$ is the distance, and $\mathrm{a}_{\mathrm{ni}}$ and $\mathrm{b}_{\mathrm{ni}}$ are the $i^{\text {th }}$ components of the input voice signal. The $\mathrm{n}$ index points to each of the five vector pairs.

$$
D=\frac{1}{5} \sum_{n=1}^{5} \sqrt{\sum_{i=1}^{1024}\left(a_{n i}-b_{n i}\right)^{2}}
$$

The block diagram is defined in Fig.-8.

B. 0-Level DFD for AVSD

Algorithm for AVSD 


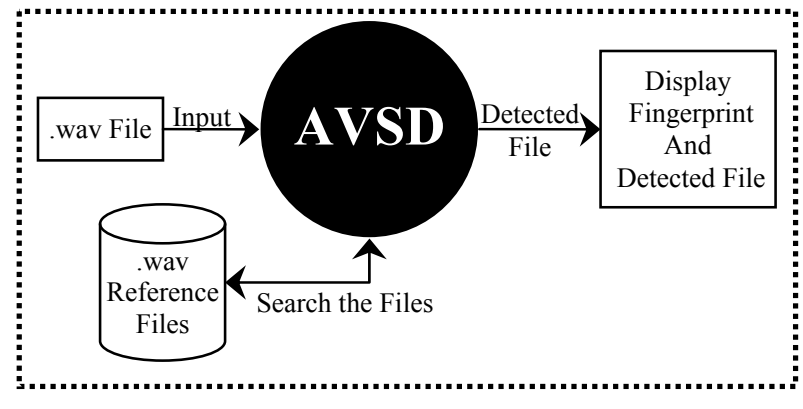

[Fig.-9: 0-Level DFD for AVSD]

The proposed algorithm for AVSD is given in following steps in which multiples .wav files are selected a reference file and fingerprint is find out for reference files which is defined from step-1 to step-12. All the data of fingerprint of reference file is recalled for voice signal detection which is defined from step-13 to step-22.

1) Load the reference files.

2) Set quantization parameters, Window length, Voice length, Threshold, FFT.

3) Decimate the voice signal.

4) Quantize the voice signal.

5) Initialize the pointer to detect the beginning of the word.

6) Initialize the average window.

7) Go through the voice until the difference between the averages of two adjacent windows is significant.

8) Store the detected voice signal.

9) Add FFT for each file together.

10) Average the FFT.

11) Display the fingerprint of voice signal.

12) Save data for voice detection phase of the algorithm.

13) Load data for voice signal detection.

14) Define maximum acceptable distance.

15) Initialize the distance variable, FFT Vector.

16) Load the file which will detect from reference file.

17) Decimate the signal.

18) Quantize the signal.

19) Initialize average window.

20) Go through the voice until the difference between the averages of two adjacent windows is significant.

21) Compute the distance from fingerprint.

22) Display the result (word is detected or not).

23) Display the fingerprint of voice signal.

\section{Proposed Result}

As per proposed algorithm, AVSD will automatically detect constitutional and unconstitutional voice signal for male and female voice signal .wav voice signal. It will illustrate fake voice signal for both mono and stereo .wav file for more than 15 words. For AVSD, I decide the length of voice signal is 1.024 seconds. AVSD will support both mono and stereo .wav file but if recorded .wav file will compressed .wav file format then processing of AVSD will be better that will take very less time for execution. For this problem, VSC (Voice Signal Compression) is already developed and published also which compress $50 \%$ of the source .wav file in same extension.

The AVSD is applicable for pervasive computing.

\section{REFERENCES}

[1] Shaleena Jeeawoody, "Voice Analysis and Recognition as a Car Theft Deterrent", California State Science Fair 2008, Project No: J1307, Ap2/08.

[2] http://www.USC.edu/CSSF/Current/Projects/J1307.pdf

[3] Khalid Saeed, "Sound and Voice Verification and Identification a Brief Review of Töeplitz Approach", Znalosti 2008, pp. 22-27, ISBN 978-80-227-2827-0.FIIT STU Bratislava, .stav informatiky a softvÈrovÈho inæinierstva, 2008.

[4] http://znalosti2008.fiit.stuba.sk/download/articles/znalosti2008-Saeed. pdf

[5] Maria Markaki, Andre Holzapfel, Yannis Stylianou "Singing Voice Detection using Modulation Frequency Features", Computer Science Department, University of Crete, Greece.

[6] http://www.sapa2008.org/papers/122.pdf

[7] Vijay K Chaudhari, Dr. R.K. Singh, Dr. Dinesh Varshney, Shiv Kumar, "A New Approach for Voice Spectrum Analysis (VSA) Suitable for Pervasive Computing Using Matlab”, 2009 International Conference on Software Technology and Engineering, Chennai-India on July 24 26, 2009, ISBN: 978-9-8142-8724-1, Volume No.: 22, Page No.: 390, Published by the World Scientific, Chennai, India, (C) Copyright 2009 IACSIT.

[8] http://www.iacsit.org/proceeding/icste09.toc.pdf

[9] http://www.worldscibooks.com/etextbook/7533/7533_toc.pdf

[10] Andress Holzinger with assistance from G. Searle, "Multimedia Basics", and Volume 1: Technology, ISBN: 81-7008-243-9, Firewall Media Publication.

[11] Johan F. Koegel Buford, “Multimedia System”, ISBN: 81-7808-162-8, Tenth Indian Reprint, Pearson Publication.

[12] Mohamed Cherif Amara Korba, Djemil Messadeg, Rafik Djemili, Hocine Bourouba, "Robust Speech Recognition Using Perceptual Wavelet Denoising and Mel-frequency Product Spectrum Cepstral Coefficient Features", Proceeding in International Conference Informatica-32 (2008) 283-288.

[13] http://www.informatica.si/PDF/32-3/06_Robust\%20Speech\%20Reco gnition $\% 20$ Using\%20Perceptual\%20Wavelet $\% 20$ Denoising.pdf

[14] Pavel Rajmic and Jan Vlach, "Real-Time Audio Processing Via Segmented wavelet Transform" Proc. of the 10th Int. Conference on Digital Audio Effects (DAFx-07), Bordeaux, France, September 10-15, 2007.

[15] http://dafx.labri.fr/main/works/p055.pdf

[16] Bastiaan Kleijn , "Speech Signal Processing", Speech, Music and Hearing TMH/KTH Annual Report 2001.

[17] www.speech.kth.se/annualreport/2001/11-14tsb.pdf

[18] Math Work on MatLab, Webpage, 2009-10.

[19] http://www.mathworks.com

[20] Amara Graps, Institute of Electrical \& Electronics Engineers, "An Introduction to Wavelet", Published by the IEEE Computer Society, 10662 Los Vaqueros Circle, Los Alamitos, CA 90720, USA, Vol. 2, and Num. 2.

[21] http://www.amara.com

[22] Sorin Dusan, James Flanagan, Amod Karve, Mridul Balaraman, "Speech Coding Using Trajectory Compression and Multiple Sensors", Center for Advanced Information Processing (CAIP)- Rutgers University, Piscataway, NJ, U.S.A.

[23] http://www.caip.rutgers.edu/ Sdusan/dfkb_icslp2004.pdf

[24] Tsung-Han Tsai, Yi-Wen Wang, Shih-Way Hung “An MDCT-Based psychoacoustic model co-processor design for MPEG-2/4 AAC audio encoder" Proceeding in the 7th International Conference on Digital Audio Effects (DAFx'04), Naples, Italy, October 5-8, 2004.

[25] http://www.mp3-tech.org/programmer/docs/P_335.pdf

[26] N. Ruiz Reyes, M. Rosa Zurera, F. López Ferreras, D. Martínez Muñoz, J.M. Villafranca, “ A New Cost Function To Select The Wavelet Decomposition For Audio Compression”, SPAIN.

[27] N. Ruiz Reyes, M. Rosa Zurera, F. Lopez Ferreras, D. Martínez Munoz, "A New Perceptual Entropy-based Methods to Achieve Signal Adpted Wavelet Tree in A low Bit Rate Perceptual Audio Coder", SPAIN

[28] http://195.134.67.70/eurasip/Proceedings/Eusipco/Eusipco2000/sessi ons/FriAm/PO1/cr1567.pdf

[29] N. Ruiz Reyes, M. Rosa Zurera, F. Lopez Ferreras, P. Jarabo Amores, P. Vera Candeas, "On the Coding Gain of Dynamic Huffman Coding Applied to a Wavelet Based Perceptual Audio Coder", Escuela Universitaria Politénica 23700 Linares - Jaén (SPAIN).

[30] http://www.mp3-tech.org/programmer/docs/page335_id073.pdf

[31] Grigor Marchokov, Atanas Gotchev, Zdravko Nikolov, "A Wavelet-Packet Based Speech Coding Algorithm" Ministry of Education and Science, National Science Council, under the Grand No: MU-I-003/96, Proceeding in 2001. Sofia.

[32] http://www.iit.bas.bg/PECR/51/26-37.pdf 
[33] Caroline Chaux, Laurent Duval2 and Jean-Christophe Pesquet,"2D Dual-Tree M-Bandwavelet Decomposition", Universit de Marne-la-Vall¥ee, Champs-sur-Marne, France

[34] http://www-syscom.univ-mlv.fr/ chaux/articles/icassp05.pdf

[35] S.Manikandan, "Speech Enhancement Based On Wavelet De-noising", Academic Open Internet Journal, Valume17, 2006, ISSN-1311-4360.

[36] http://www.acadjournal.com/2006/V17/part1/p7/wavelet.pdf

[37] George Tzanetakis, Georg Essl, Perry Cook, "Audio Analysis using the Discrete Wavelet Transform", Computer Science Department *also Music Department.

[38] http://soundlab.cs.princeton.edu/publications/2001_amta_aadwt.pdf

[39] Claudia Schremmer, Thomas Haenselmann , Florian Bömers, "Wavelets In Real-Time Digital Audio Processing: A Software For Understanding Wavelets In Applied Computer Science", Department of Praktische Informatik IV, University of Mannheim, Germany

[40] http://www.informatik.uni-mannheim.de/pi4/publications/Schremmer 2000a.pdf

[41] Chalermchon Satirapod, Clement Ogaja, Jinling Wang and Chris Rizos, "An Approach to GPS Analysis incorporating Wavelet Decomposition", School of Geomatic Engineering, The University of New South Wales, Sydney NSW 2052, AUSTRALIA. http://www.gmat.unsw.edu.au/snap/publications/satirapod_etal2001d. pdf

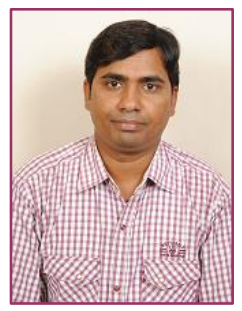

Shiv Kumar received Diploma (Lather Technology Branch) from Govt. Lather Institute, Agra (U.P.)/ Board of Technical Education, Lucknow (U.P.)-India in year 2000. He worked as a Tanner in Ajaction Lather Punjab Ltd. (Punjab)-India in during 2000 to 2001. After that he completed B. Tech. (Information Technology Branch) from Bhagwant Institute of Technology, Muzaffarnagar (U.P.)/ Uttar Pradesh Technical University-Lucknow (U.P.)-India in year 2004, and M. Tech. (Honors, Information Technology Branch) from Technocrat Institute of Technology (TIT), Bhopal (M.P.)/ Rajeev Gandhi Technical University, Bhopal (M.P.)-India in year 2010. Now he is Pursuing Ph.D. (Computer Science \& Engineering Branch) from Banasthali University, Tonk (Rajasthan). He worked as a lecturer in the department of C.S.E. / I.T. at Chouksey Engineering College (CEC), Bilaspur (C.G.)-India from Dec-2004 to April-2006, and at Shri Shankaracharya College of Engineering and Technology (SSCET), Bhilai (C.G.)-India in between May-2006 to August-2006. Now he is working as an Asst. Professor in the Department of Information Technology at Technocrat Institute of Technology (TIT), Bhopal (M.P.)-India since 07 Nov. 2006. His research area interest includes Voice Signal Compression, Tonality Computation of Voice Signal, Image Processing, Spectrum Analysis of Voice Signal, Voice Recognition, Pitch Computation of Voice Signal, Formant Analysis of Voice Signal, Loudness Analysis of Voice Signal, and Voice Signal Detection. He is having 16 papers/articles in International Journals (IJCEE, IJET, CiiT International Journal of Digital Signal Processing, CiiT International Journal of Image Processing, CiiT International Journal of Programmable Device Circuits and Systems, CiiT International Journal of Biometrics and Bioinformatics, IJCEA, IJCM, IJES, IEEE Xplore Digital Library, IEEE CS Digital Library, ACM Digital Library, and WSP), 07 papers in International Conferences, 8 papers in National Conferences, 05 International/National Seminar, and 01 Text Book (Concept of Operating System). He is reviewer for 04 International Journals (Journal of Electrical and Engineering and Research (JEEER)-Victoria (Island), Journal of Computer and Communication (JCC)-USA, Journal of Computer Technology and Application (JCTA)-USA, International Journal of Computer Theory and Engineering (IJCTE)-Singapore). He is senior member of IACSIT \& IAENG. He has given great contribution in the field of Voice Signal Analysis. He believes in only and only work.

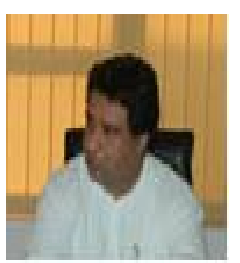

Prof. Aditya Shastri is currently serving as Vice-Chancellor of Banasthali Vidyapith and discharging the duties as Chief Executive Officer and Chief Academic Officer of the University. He was born on 4 June, 1963 in a family of academics where higher education and social service was valued the most. His grandfather Pandit Hiralal Shastri was the First Chief Minister of Rajasthan, a member for the constituent assembly and founded Banasthali Vidyapith in 1935 along with Smt. Ratan Shastri, a recipient of Padma Bhusan and the Jamnalal Bajaj Awards for her contribution to Banasthali. Aditya Shastri showed a great deal of promise in academics from the very early age and secured $4^{\text {th }}$ rank in the Higher Secondary examination of the Board of Secondary Education, Rajasthan. He was also a rank holder in the Secondary examination securing $8^{\text {th }}$ position. On completion of his education from BITS, Pilani in 1984 from where he received M.Sc.(Tech.) Computer Science and M.Sc.(Hons.) Mathematics, he went to USA for further studies and earned Master's degree in Mathematics from SUNY, Stony Brook, before moving to Massachusetts Institute of Technology (MIT) from where he completed his Ph.D in 1990. After serving the Tata Institute of Fundamental Research (TIFR) for one year, he moved to Banasthali where he has remained ever since. His initial years at Banasthali were mainly devoted to developing IT and Computer Science activities which culminated in the establishment of Apaji Institute of Mathematics and Applied Computer Technology (AIM \& ACT). During this period he has held several visiting appointments in the UK, France and Japan; most notably, he was selected as a Marie-Curie Fellow by DST in 1994-95 and spent that year in Universite Du Maine, France. This fellowship is given to only one scientist every year. He was also a Royal Society Exchange Visitor to the University of Nottingham in 1993 and a research staff member at IBM-India Research Lab. from 1999-2000. He has published over 50 research papers and has authored five text-books. He has carried out a number of research projects for various funding agencies. Most notably he is the Principle Investigator of a project from Ministry of Information Technology titled "Socio-economic development of Rajasthan through IT" valued at Rs. 1.00 crore. His research interests include Discrete Mathematics, Combinatorics, Graph Theory, Theory of Computation, (parallel) algorithms, E-commerce and Mobile Computing. Of late, he is spending much of his time and energy for the overall development of Banasthali. Over the last few years the Vidyapith has attained self-reliance through some bold initiative spearheaded by him such as the Jaipur campus, off-shore centers and massive expansion of all activities of the Vidyapith. The University has recently been accredited with 'A' grade by NAAC, Govt. of India and attracts more number of applications than ever before.

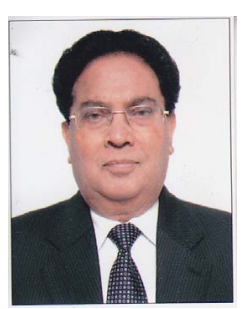

Prof. R.K.Singh received his Ph.D.degree from the Banaras Hindu University in 1969 for the Development of New Three-Body Force Shell Model for Ionic Solids. He pursued higher studies at the University of Edinburgh (U.K.) as a Commonwealth Fellow. As an excellent academic achiever with significant contributions in Science and Technology, Prof. Singh is the first recipient of Dr. K.N. Katju Award for Sciences, presented by HE President of India in 1984 for outstanding contributions in Condensed Matter Physics. Prof Singh has published 641 Research Papers (out of which 277 are in International Journals and 364 are in Conf. Proceedings) including 10 exhaustive Critical Reviews in contemporary areas and 9 Books (edited). He has visited several countries (UK, USA, Canada, Italy, Japan, Korea, Hong Kong, Singapore, Malaysia, Cyprus, Eqypt, China, Mauritius, etc.) repeated number of times to deliver lectures as Visiting Professor and participate and chair and deliver invited talk in International Conferences/ Symposium. During his research endeavor, he has supervised $60 \mathrm{Ph} . \mathrm{D}$. Scholars. The research interest of Prof. Singh spans over a wide spectrum of Condensed Matter Physics and Materials Science, which includes Nano-materials, Superconductivity, CMR materials, Multiferroic Manganites, Phonon Dynamics, Phase Transition, Orientationally Disordered Materials, Diluted Magnetic Semiconductors, Plastic Crystals and many other Electronic Ceramics. His pioneering work on many body interactions and a comprehensive review on the said topic in Physics Reports 85 (1982) 259-401 and published a Paper in Proceedings of Royal Society (London) A349 (1976) 289-309, which where were heralded by the worldwide scientific community with great appreciation. In addition to his outstanding work on the condensed matter physics, he has explored experimentally several properties of materials, particularly, high temperature superconductors. His innovative ideas are implemented through a well established Superconductivity Research Laboratory, established by him at Bhopal and also through several collaborative programmes. As an Acclaimed Institution Builder. Prof. Singh established the Department of Physics as its Founder Professor and Head at Barkatullah University, Bhopal from 1984-95 and got sanctioned and implemented Superconductivity, Special Assistance and Electronics Programmes from the University Grants Commission. As Hon. Director, he established a Centre for Science and Technology Development Studies at MP Council of Science and Technology. As Founder Director, he established the Computer Centre and Computer Science Department. He was also Head of the University Science Instrumentation Centre at Barkatullah University, Bhopal from 1984 -95. Prof. R.K. Singh has been the Vice Chancellor of Guru Ghasidas University, Bilaspur for two terms (from June 22, 1995 to Jan 22, 2002), Prof. Singh introduced 45 courses and established 8 new Institutes (namely, Institutes of 
Technology, Pharmacy, Rural Technology, Biotechnology, Computer Applications, Information Technology, Distance Education, and Chhattisgarh Institute of Medical Sciences for MBBS course), besides strengthening the existing Institutes and Departments in the University, and raised the annual budget from 3.00 Crores to 36.00 Crores from 1995-2002. Prof. R.K. Singh has been the Vice Chancellor of M. P. Bhoj (Open) University, Bhopal (from January 23, 2002 to January 4, 2005) with his wide experience and vision of establishing an Institute of Distance Education at Guru Ghasidas University. He participated in the conferences of Asian Association of Open Universities Organized by IGNOU (2001) and Korea (2002). He planned and introduced 50 new professional and job oriented courses of distance education, particularly for the students from reach to unreach. Gyanvani FM band channel was launched in Bhopal in September 2002 in collaboration with IGNOU and DEC for mass literacy mission. Besides, he got established the Institutes of Basic Sciences, Information Technology, General Education and the Centre for Information and
Communication Technology (ICT) for imparting online/e-learning courses in Management/Computer/Special Education. Prof R K Singh has been the Senior most Vice Chancellor of Madhya Pradesh Universities. He was elected the Member of the Executive Council of the Association of Commonwealth Universities for 2002-03 and 2003-04. He is the elected Vice President of the International Academy of Physical Sciences for 2004-06. He was elected as Vice President, Association of Indian Universities in November, 2004. Prof. Singh has been working as Dean, Academic Affairs and Director, University Centre for Distance Learning, Chaudhary Devi Lal University, Sirsa (Haryana), from Sept 2006- Nov 2007. Prof R.K. Singh has been the Pro-Vice Chancellor, MATS UNIVERSITY, RAIPUR (C.G.). Prof. Singh is Emeritus Professor of Physical Sciences at Indian Institute of Information Technology, Allahabad. Prof R.K. Singh is working as Vice Chancellor, ITM UNIVERSITY, GURGAON (HRY.) 122017 from January 2010. 